\title{
Bias-induced impedance effect of the current-carrying conductors
}

\author{
Sioma Baltianski ${ }^{1}$ \\ ${ }^{1}$ Wolfson Dept. of Chemical Engineering, Technion - Israel Institute of Technology, Haifa, Israel
}

\section{ABSTRACT}

The paper presents the previously unstudied properties of current-carrying conductors utilising impedance spectroscopy. The purpose of the article is to present discovered properties that are the significant context of impedance research. The methodology is based on the superposition of test signals and bias affecting the objects under study. These are the main results obtained in this work: the studied objects have an additional low-frequency impedance during the passage of an electric current; the bias-induced impedance effect $\left(Z_{\mathrm{BI}}{ }^{-}\right.$ effect) is noticeably manifested in the range of $0.01 \mathrm{~Hz} \ldots 100 \mathrm{~Hz}$ and it has either capacitive or inductive nature or both types, depending on the bias level (current density) and material types. The experiments in this work were done using open and covered wires made of pure metals, alloys, and non-metal conductors, such as graphite rods. These objects showed the $Z_{B I}$-effect that distinguishes them from other objects, such as standard resistors of the same rating, in which this phenomenon does not occur. The $Z_{B I}$-effect was modeled by equivalent circuits. Particular attention is paid to assessing the consistency of experimental data. Understanding the nature of this effect can give impetus to the development of a new type of instrument in various fields.

\section{Section: RESEARCH PAPER}

Keywords: bias-induced impedance; impedance spectroscopy; $Z_{\mathrm{BI}}$-effect

Citation: Sioma Baltianski, Bias-induced impedance effect of the current-carrying conductors, Acta IMEKO, vol. 10, no. 2, article 13, June 2021, identifier: IMEKO-ACTA-10 (2021)-02-13

Section Editor: Giuseppe Caravello, Università degli Studi di Palermo, Italy

Received January 18, 2021; In final form April 15, 2021; Published June 2021

Copyright: This is an open-access article distributed under the terms of the Creative Commons Attribution 3.0 License, which permits unrestricted use, distribution, and reproduction in any medium, provided the original author and source are credited.

Corresponding author: Sioma Baltianski, e-mail: cesema@technion.ac.il

\section{INTRODUCTION}

The study of various physical objects using impedance spectroscopy under applied dc bias is widespread. These objects may be of different physical nature: semiconductor structures [1], electroceramic structures [2], [3], electrochemical objects [4], etc. In all cases, the external offset sets the operating point in the vicinity of which the impedance measurements are made. The set offset (bias) makes it possible to tie the parameters obtained from impedance measurements to the physical state of the object under study.

The idea to investigate the current-carrying conductors under the influence of bias arose because of a misunderstanding of the behavior of a fairly simple object as a load in the process of testing the different impedance meters. The first research results were published in [5]. Possible measurement errors were checked in various ways, and literature sources describing the detected effects were not found.

This work relates to impedance spectroscopy for several reasons. On the one hand, based on the described phenomena, sensitive elements can be created that require the use of impedance spectroscopy as a method for extracting informative parameters. On the other hand, it is a challenge to build more sensitive impedance meters (with an appropriate offset function) that allow obtaining reliable data under relatively difficult measurement conditions: low frequency and high tangent of the loss angle. The goal of this work is to reveal the discovered properties, which are important in the context of impedance research.

Low-frequency impedance spectroscopy was used as a method. The complex conductivity function and its determination by approximating experimental data utilising different models serve as theoretical basis [6]-[8].

The impedance of current-carrying conductors is well known and described in the literature [9]. As an example, we give the behaviour of the impedance of a silver conductor $0.5 \mathrm{~m}$ long and $0.25 \mathrm{~mm}$ in diameter. The initial experiment in Figure 1 without bias (at $V d c=0 \mathrm{~V}$ ) represents a typical behaviour of the real and imaginary parts of the impedance in the frequency range $1 \mathrm{MHz}$ .. $100 \mathrm{mHz}$. First, we use the full frequency range to verify the processes. Later, we will be interested in events only in the low 


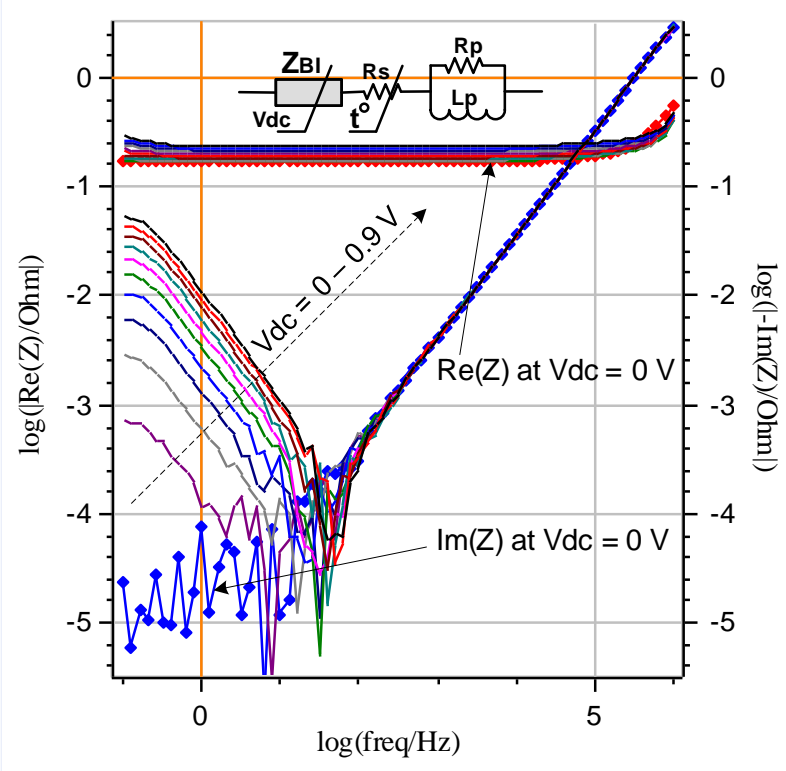

Figure 1. $\operatorname{Re}(Z)$ and $\operatorname{Im}(Z)$ of the silver wire at $V d c=0 \mathrm{~V} \ldots 0.9 \mathrm{~V}$ with bias step $0.09 \mathrm{~V}$; length $500 \mathrm{~mm}$ and diameter $0.25 \mathrm{~mm}$; frequency range $1 \mathrm{MHz} \ldots$ $0.1 \mathrm{~Hz}$.

frequency part of the spectrum. The initial and all subsequent potentiostatic experiments were carried out at the amplitude of the test signal $V a c=10 \mathrm{mV}$. This signal corresponds to a small signal approach. The view of graph results is quite trivial at zero bias. Three approximate frequency domains can be distinguished in this graph: the high frequency region $(\mathrm{HF})$ of the spectrum $f=1 \mathrm{MHz} \ldots 100 \mathrm{kHz}$; the mid frequency region (MF) $f=100 \mathrm{kHz} \ldots 100 \mathrm{~Hz}$ and the low frequency region (LF) $f=100 \mathrm{~Hz} \ldots 0.1 \mathrm{~Hz}$.

In the $\mathrm{HF}$ region there is an increase of a real $\operatorname{Re}(Z)$ and imaginary $\operatorname{Im}(Z)$ part of impedance with increasing frequency. This part is well described by a parallel connection of resistance $R p$ and inductance $L p$ (Figure 1).

In the $M F$ region, a constant value of $\operatorname{Re}(\mathrm{Z})$ is observed. Series resistance Rs must be added to the model. A linear decrease in the imaginary component occurs with a decrease in frequency (log-log scale). The relative noise level increases at the same time. This noise is natural and associated with the measuring system capabilities. The LF part of the spectrum at $V d c=0 \mathrm{~V}$ demonstrates the constancy of $\operatorname{Re}(Z)$ and the strong noise of $\operatorname{Im}(Z)$. This area is not informative for interpretation using the imaginary part of the impedance. Measurements were made using a Faraday cage to improve the signal-to-noise ratio mainly for the imaginary impedance component.

In this simple model, the specific resistance of the conductor determines the series resistance Rs. Mainly the length of the conductor determines the inductance $L p$. The parallel resistance $\mathrm{R} p$ that is connected to the inductance characterises the active loss in the conductor due to the skin effect at high frequencies. The experimental values at zero bias correspond to the expected values and are quite common.

The situation changes significantly when measurements are carried out under bias. The experimental characteristics are shown in the same Figure 1 at biases $V d c=0.09 \mathrm{~V} \ldots 0.9 \mathrm{~V}$. The increment of bias was $0.09 \mathrm{~V}$, and the measuring test signal was the same, namely $V a c=10 \mathrm{mV}$. The HF and the MF imaginary part of the impedance do not change with bias. However, the LF part changes considerably. This response can be reflected by including an additional non-linear impedance $Z_{B I}$ (Figure 1). A corresponding increase of the real part of the impedance occurs in the considered region, which meets the Kramers-Kronig relationships [6]. Besides, we observe a monotonic change in the real component of the impedance in the entire frequency range (the model element Rs). This change is caused by a shift in the temperature of the conductor due to biases. The model indicated in Figure 1 is intuitive, but it well describes and fits the object under study in the specified frequency range.

The bias phenomenon is sometimes difficult to detect because experimental data are often on the limit of the sensitivity of measuring instruments, namely, limitations on phase measurement with a high value of the loss tangent. Potentiostat/Galvanostat Biologic SP-240 (BioLogic Science Instruments) was used as a measuring instrument. In doubtful cases, data were verified using the Potentiostat/Galvanostat Gamry Reference 3000 (Gamry Instruments). A Biologic's contour plot defines an error not more than $0.3 \%$ and $0.3^{\circ}$ in the desired measuring range [10]. Thus, the experimental data in Figure 1 is reliable. Moreover, in this case, we are interested in relative changes in the impedance components.

A homemade four-wire sample holder was used to connect the samples under test (SUT), as shown in Figure 2. Electrode designation is taken from the manual [10]. This sample holder gives accurate measurement of low-resistance objects as well as negligible influence of contact phenomena.

In addition to the devices used in this work (Biologic and Gamry), which are based on Frequency Response Analyzer, also the devices of the Lock-in Amplifier type can be used (see for example [11]). We used standard not wire wound resistors as references to verify measuring results and estimate artifacts. This phenomenon was not observed when dealt with standard resistors of the same rating as SUT.

The impedance change in the LF region can be caused not only by bias using direct current but also by alternating current by a large amplitude test signal at zero bias. It should be emphasised that in this work, we use a small signal approach in which a change in the LF impedance is not observed at zero bias. Thus, the occurrence of the additional impedance in the LF region will be determined solely by the level of bias. This physical phenomenon is named here as a bias-induced impedance $\left(\mathrm{Z}_{\mathrm{BI}^{-}}\right.$ effect).

In Section 2 we will systematise the experimental results. Section 3 is devoted to the interpretation of experimental results

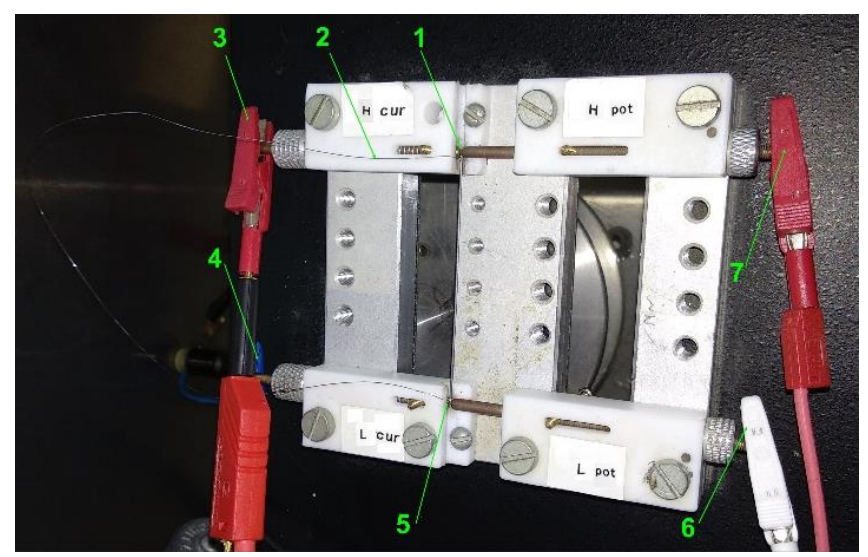

Figure 2. Four-Wire Sample Holder. 1 - High force-and-sense Kelvin clip; 2 Sample Under Test (wire); 3 - Working Electrode; 4 - Counter Electrode; 5 Low force-and-sense Kelvin clip; 6 - Reference Electrode; 7- Working Sence Electrode. 
using electrical models. Section 4 discusses significant differences in impedance behavior of open and covered objects. Special attention is paid to checking the consistency of experimental data: this is outlined in the Section 5. Section 6 discusses and proves the main hypotheses that explain the revealed effect. Finally, the main results are presented in the concluding section.

\section{SYSTEMATISATION OF THE EXPERIMENTAL RESULTS}

We studied pure metals: nickel, copper, silver, tungsten, platinum, gold; alloys: constantan, nichrome, manganin; nonmetals - graphite rods. Although the frequency scan started from $1 \mathrm{MHz}$ toward low frequencies, the analysis of the results was carried out only for the LF part of the spectrum, where the $\mathrm{Z}_{\mathrm{BI}^{-}}$ effect manifested.

According to the type of $\mathrm{Z}_{\mathrm{BI}-\text { effect, all studied materials were }}$ grouped into three categories: (i) the $\mathrm{Z}_{\mathrm{BI}}$-effect has a capacitive nature, (ii) - an inductive nature, and (iii) - mixed when both types of reactance occur. Table 1 summarises the properties of the investigated materials. Below are the experimental characteristics of one of the representatives of each group.

\subsection{Pure metals}

We find significant changes in the behaviour of the imaginary part of the impedance after a critical frequency of about $30 \mathrm{~Hz}$ (resonance point) when applying bias (Figure 1). The inductive nature of reactance sharply changes to a capacitive nature from this point to the direction of LF. We observe a monotonic change in the imaginary and real component of the impedance, depending on the applied bias.

Changes in the real part of impedance in the mid frequency region also occur; however, this is due to a change in the temperature of the conductor upon bias. For example, with a maximum bias of $0.9 \mathrm{~V}$ for this experiment and a conductor resistance of about $0.23 \Omega$, the current flowing through the conductor will be approximately 3.9 A. The power dissipation will be approximately $3.5 \mathrm{~W}$, which will lead to certain heating of the conductor and consequential increase in its resistance. Figure 3 shows a Nyquist graph of the LF part of the same experiment, which is shown in Figure 1.

At mid and high frequencies, there is no change in the behaviour of the imaginary component of the impedance under the influence of bias. Henceforward, we will limit visualisation within the LF part of experimental data in the form of Nyquist plots. Similar in appearance, but numerically different in values, the characteristics were obtained in studies of other pure metals: nickel, copper, tungsten, platinum, and gold.

\subsection{Alloys}

The impedance characteristics of alloys as a function of bias differ from pure metals. Manganin demonstrated the inductive nature of reactance at moderate bias. In our nichrome and constantan samples, the $\mathrm{Z}_{\mathrm{BI}}$-effect had both capacitive and inductive reactance. The nature of the reactance depends on the level of bias.

Table 1. Systematisation of the investigated materials by the nature of ZBIeffect.

\begin{tabular}{cccc}
\hline $\begin{array}{c}\text { Type of } \\
\text { conductors }\end{array}$ & $\begin{array}{c}\text { Pure } \\
\text { metals }\end{array}$ & Alloys & $\begin{array}{c}\text { Non-metals } \\
\text { (Graphite) }\end{array}$ \\
\hline $\begin{array}{c}\text { Nature of } \\
\mathrm{Z}_{\mathrm{B} \text {-effect }}\end{array}$ & Capacitive & $\begin{array}{c}\text { mixture: } \\
\text { Capacitive and } \\
\text { Inductive }\end{array}$ & Inductive \\
\hline
\end{tabular}

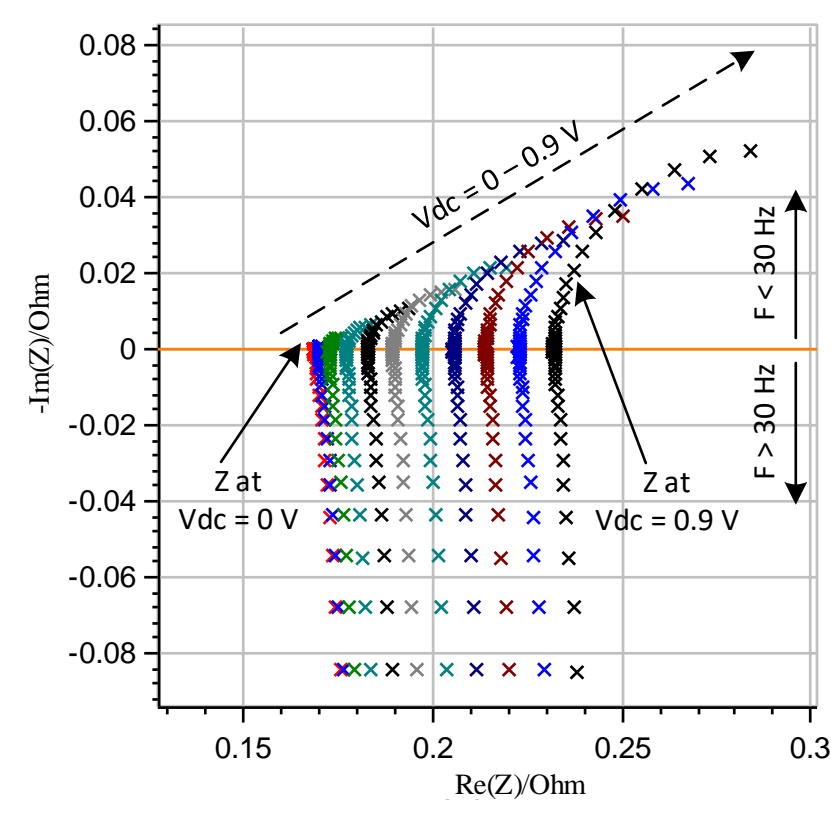

Figure 3. Nyquist plot of silver wire at $V d c=0 \mathrm{~V} \ldots 0.9 \mathrm{~V}$ with bias step $0.09 \mathrm{~V}$ length $500 \mathrm{~mm}$ and diameter $0.25 \mathrm{~mm}$.

As an example, studies of a nichrome sample with a diameter of $0.1 \mathrm{~mm}$ and a length of $57 \mathrm{~mm}$ are presented in Figure 4.

The experiment was carried out using a bias in the range of $2.8 \mathrm{~V} \ldots 8.5 \mathrm{~V}$ in increments of $0.1 \mathrm{~V}$. The data were taken in the frequency spectrum $1 \mathrm{MHz} \ldots 0.1 \mathrm{~Hz}$, but only the range of interest is presented here: $100 \mathrm{~Hz} \ldots 0.1 \mathrm{~Hz}$.

Three areas of bias were identified. In the bias of $2.8 \mathrm{~V} \ldots$ 4.6 V the capacitive nature of the reactance was observed. In the

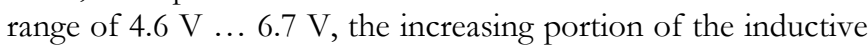
nature of the reactance added to the decreasing portion of the capacitive nature of the reactance. With a subsequent increase in bias, the reverse process occurs. Also, in the bias range of $6.7 \mathrm{~V}$ .. $8.5 \mathrm{~V}$, the capacitive nature of the reactance was again

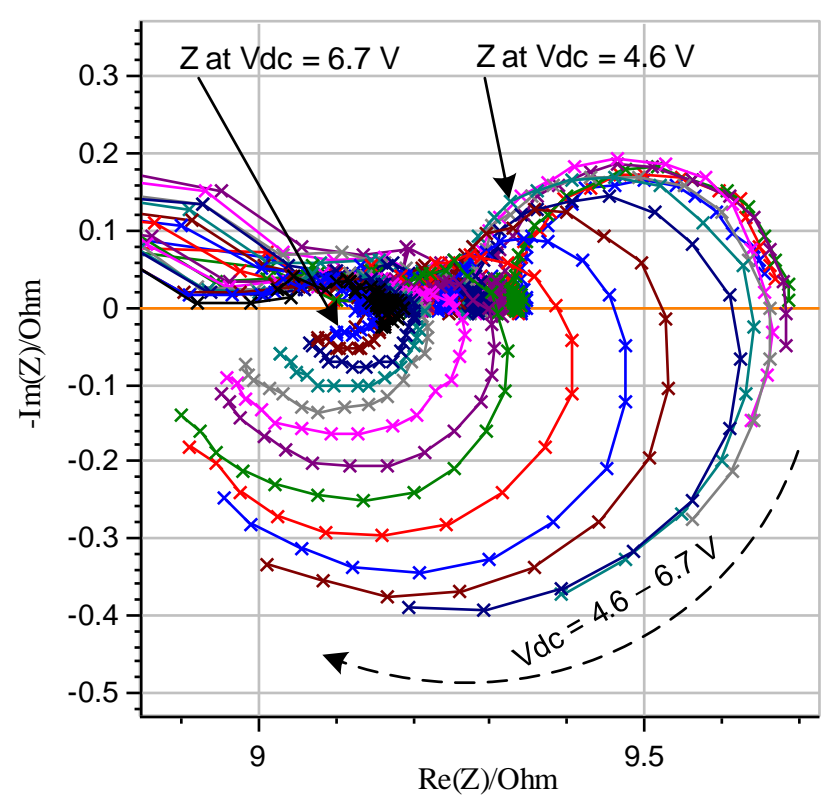

Figure 4. Nyquist plot of nichrome wire at $\mathrm{Vdc}=4.6 \mathrm{~V} \ldots 6.7 \mathrm{~V}$ with bias step $0.1 \mathrm{~V}$; length $57 \mathrm{~mm}$ and diameter $0.1 \mathrm{~mm}$. 


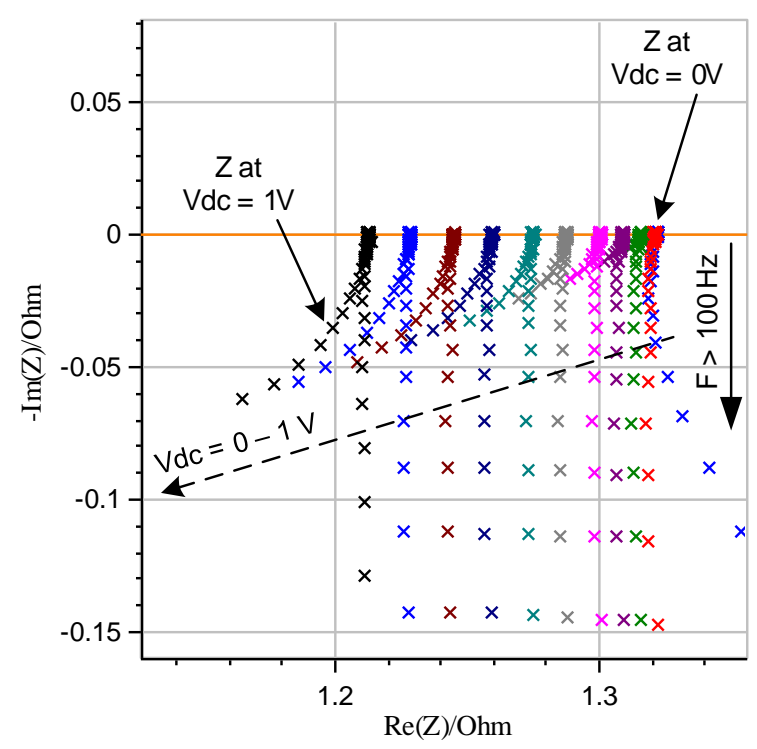

Figure 5. Nyquist plot of graphite rod at $\mathrm{Vdc}=0 \mathrm{~V} \ldots 1 \mathrm{~V}$ with bias step $0.1 \mathrm{~V}$; length $57 \mathrm{~mm}$ and diameter $0.5 \mathrm{~mm}$.

observed, the same as with a small bias. Figure 4 shows a transient state when both types of reactance are present.

\subsection{Non-metals}

Measurements were carried out on graphite rods. Samples of various diameters were investigated.

Figure 5 shows Nyquist plots of the impedance of the graphite rod $0.5 \mathrm{~mm}$ in diameter and $57 \mathrm{~mm}$ in length. The inductive nature of reactance was demonstrated over the entire range of biases.

\section{INTERPRETATION USING ELECTRICAL MODELS}

First, we consider a simple case of interpreting experimental data related to pure metals in which the $\mathrm{Z}_{\mathrm{BI}}$-effect of capacitive nature is manifested. As an example, Figure 6 presents a fitting result of the LF part of one of the experiments shown in Figure 3, specifically at bias $V d c=0.72 \mathrm{~V}$.

The fitting was carried out using the impedance model which consists of serial resistor $R s$ connected to parallel $C_{1}$ and $R_{1}$. The resistor $R s$ reflects specific resistance of the sample under test and its geometry. The resistance varies with the applied bias,

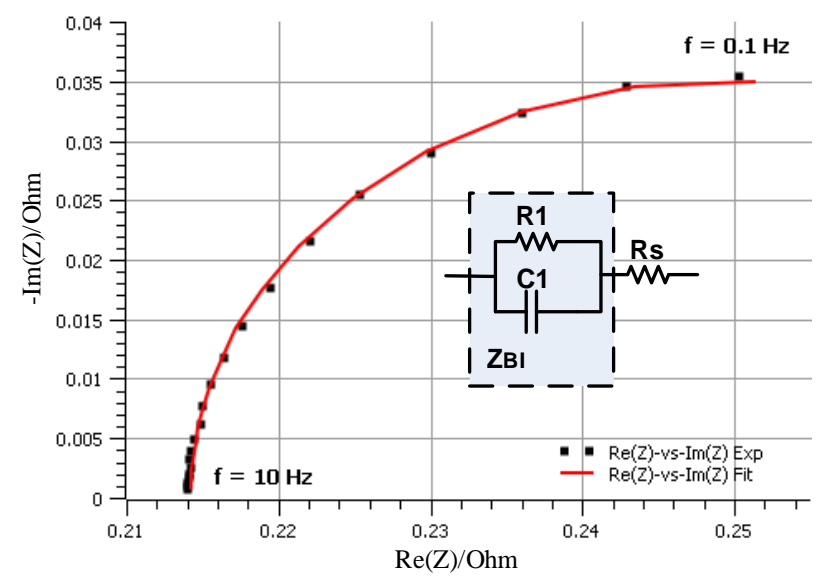

Figure 6. Fitting result of LF part of data $(f=10 \mathrm{~Hz} \ldots 0.1 \mathrm{~Hz})$. Silver wire at $V d c=0.72 \mathrm{~V}: R_{\mathrm{s}}=0.214 \Omega ; R_{1}=0.07 \Omega ; C_{1}=21.34 \mathrm{~F}$.

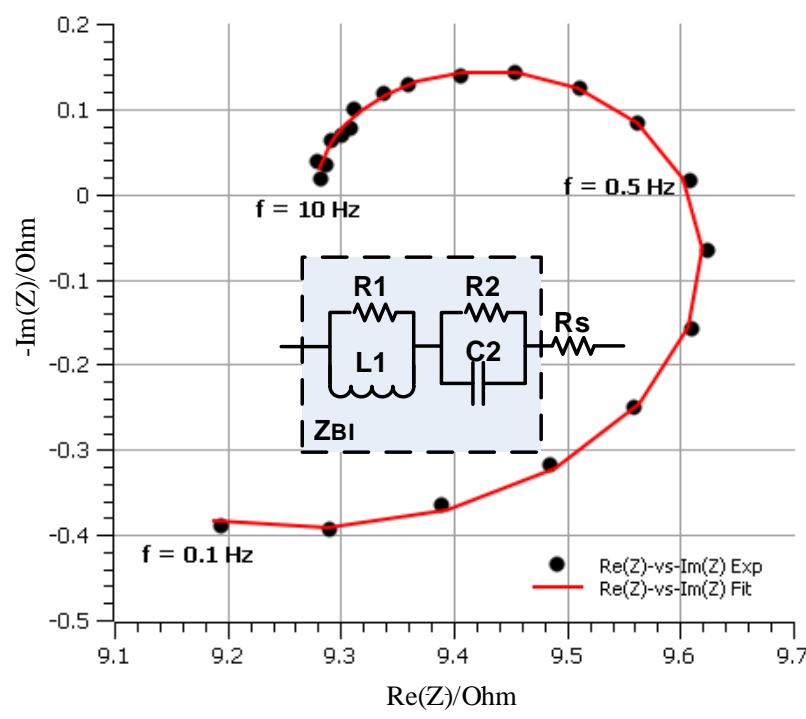

Figure 7. Fitting result of LF part of data $(f=10 \mathrm{~Hz} \ldots 0.1 \mathrm{~Hz}$ ). Nichrome wire at $\mathrm{Vdc}=5.3 \mathrm{~V}$ : Rs=8.261 $\Omega ; \mathrm{R} 1=0.622 \Omega ; \mathrm{C} 1=0.373 \mathrm{~F} ; \mathrm{R} 2=1.018 \Omega$; $\mathrm{L} 1=1.091 \mathrm{H}$.

which affects the temperature of the sample (see a right shift of characteristics in Figure 3 with increasing bias). The parallel circuit $C_{1}-R_{1}$ exactly describes the $Z_{\mathrm{BI}}$ effect. Figure 6 shows a good fitting quality.

A similar approach for fitting can be used for materials in which the $\mathrm{Z}_{\mathrm{BI}}$-effect is purely inductive (Figure 5) by using the $L R$ - circuit. The situation becomes more complicated in the case of a complex $\mathrm{Z}_{\mathrm{BI}}$-effect (Figure 4). One of the possible electrical models that satisfactorily approximate the experimental data is embedded in Figure 7.

A system function as a rational fraction [12] that corresponds to this model has the following form:

$$
Z(s)=\frac{A_{0}+A_{1} s+A_{2} s^{2}}{1+B_{1} s+B_{2} s^{2}},
$$

where $s=j 2 \pi f$ and $A_{i}, B_{i}$ are unknown coefficients.

Although the system function uniquely approximates the experimental data, its coefficients are difficult to fill with a physical meaning. It is easier to do this using circuit functions which reflect the topology of the corresponding equivalent circuits [12]. The circuit function corresponding to the model in Figure 7 is described by the following equation:

$$
Z(s)=R_{s}+\frac{R_{1}}{1+\tau_{C} \cdot s}+\frac{L_{1} \cdot s}{1+\tau_{L} \cdot s},
$$

where: $\tau_{C}=R_{1} \cdot C_{1} ; \tau_{L}=L_{1} / R_{2}$ and $R_{s}, R_{1}, R_{2}, C_{1}, L_{1}$ requested parameters from fitting.

The system function (1) covers several equivalent circuits. Figure 7 represents one of the possible implementations. The results of fitting utilising this circuit for one of the characteristics represented in Figure 4, specifically at bias $V d c=5.3 \mathrm{~V}$, are given in Figure 7. The selection of a suitable electrical model can be made empirically by iterating through the available set of models determined by the system function (1). To implement this process, experimental data can be approximated using an acceptable set of equivalent circuits and utilising available fitting programs, such as LEVM [6] or method described in [13].

Figure 8 represents the dependencies of the model parameters versus bias of the silver wire corresponding to the data shown in Figure 3. 


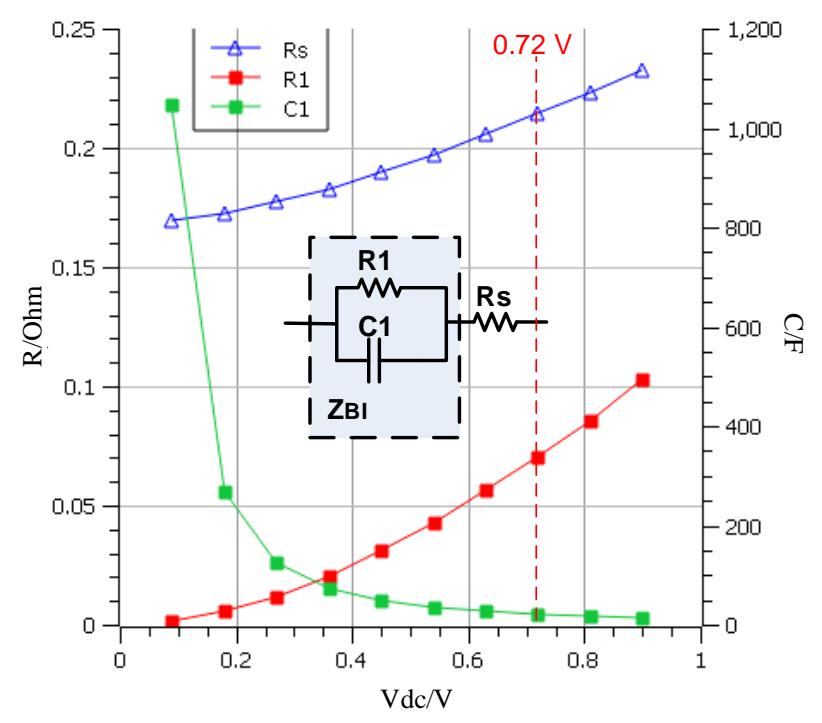

Figure 8. Model parameters of silver wire as a function of bias - refer to the experiment data in Figure 3.

The capacitance $C_{1}$ increases exponentially with decreasing bias. This leads to a decrease in the contribution of the reactive

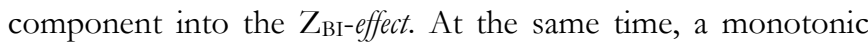
decrease in resistance $R_{1}$ is observed. As a result, at zero bias, the $\mathrm{Z}_{\mathrm{BI}}$-effect demonstrated by the vanishingly small magnitude.

The resistance $R s$ reflects a change in resistivity as a function of temperature, which in turn depends on the flowing bias current. The temperature value (it got by utilising resistivity) and power dissipation for this experiment shown in Figure 9. Similar results, but differing in values, were obtained for other pure metals.

The graphite rod model behaves quite differently as shown in Figure 10. First, the series resistance $R s$ decrease with bias due to a negative temperature coefficient (NTC). It distinguishes them from metal in which there is a positive coefficient of resistance (PTC), see Figure 8. Secondly, the inductance $L_{1}$, together with the parallel resistance $R_{1}$, decreases with decreasing bias. This nullifies the $\mathrm{Z}_{\mathrm{BI}}$-effect at zero bias.

The behaviour of model parameters for alloys is more complex and is beyond the scope of this article.

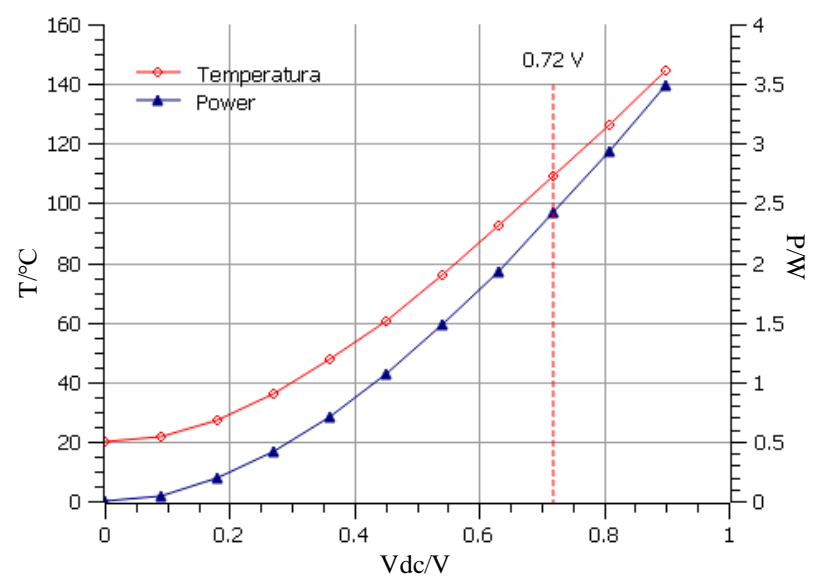

Figure 9. Power dissipation and temperature of silver wire - refer to the experiment data in Figure 3.

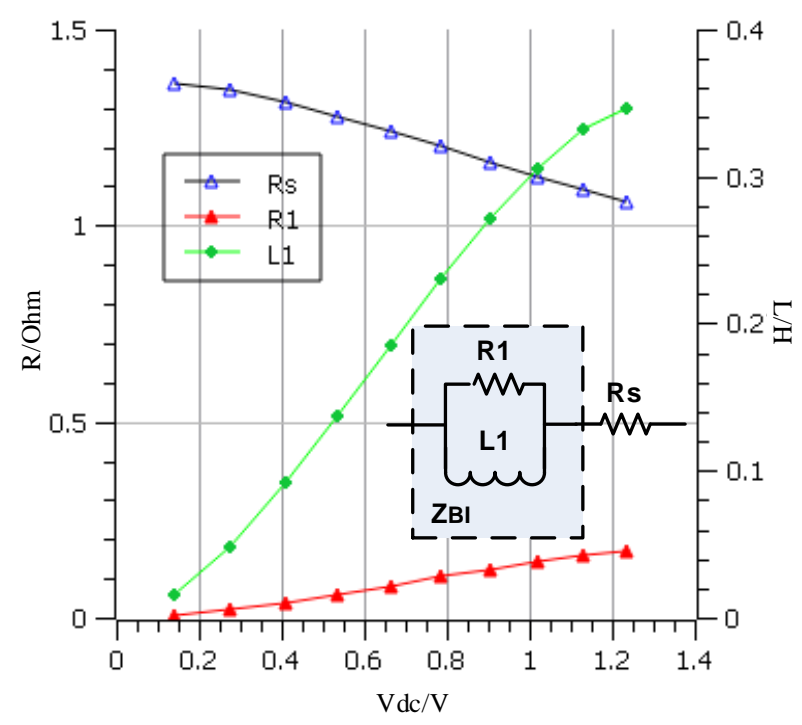

Figure 10. Model parameters of graphite rod as a function of bias - refer to the experiment data in Figure 5.

\section{THE DIFFERENCE BETWEEN OPEN AND COVERED OBJECTS}

Some of the previously investigated objects were studied using various types of cover. This is necessary to validate the hypotheses put forward to explain the occurrence of the effect as described in Section 6. The results of the study of currentcarrying conductors covered with dielectric materials are quite informative for this purpose. Here, we present the results employing a shell in the form of thin Teflon and ceramic (alumina) tubes that is quite tight adjacent to the object under study. It is necessary to expand the frequency range towards lower frequencies down to $1 \mathrm{mHz}$ to detect the effect of covering onto the impedance results. This requirement significantly increased the time of each experiment.

Figure 11 shows the real and imaginary parts of the impedance without covering. We used the galvanostatic mode of the measurement device (Biologic SP-240) and the same sample holder as previously. The full range of bias current was $I d c=$

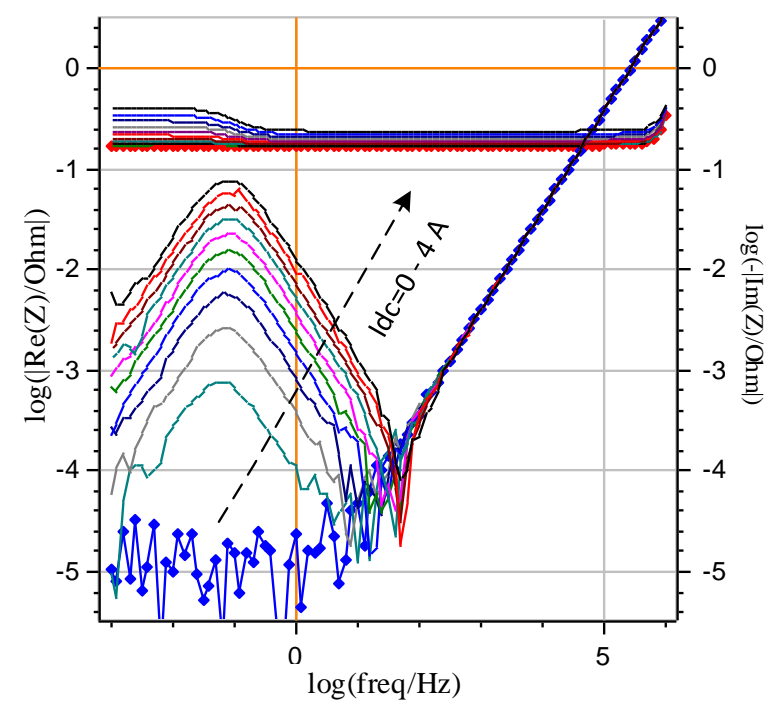

Figure 11. $\operatorname{Re}(Z)$ and $\operatorname{Im}(Z)$ of the open silver wire; galvanostatic mode at amplitude $l a c=100 \mathrm{~mA}$ and bias $I d c=0 \mathrm{~A} \ldots$.. $\mathrm{A}$ with step $0.4 \mathrm{~A}$; length 500 $\mathrm{mm}$ and diameter $0.25 \mathrm{~mm}$; frequency range $1 \mathrm{MHz} \ldots 1 \mathrm{mHz}$. 


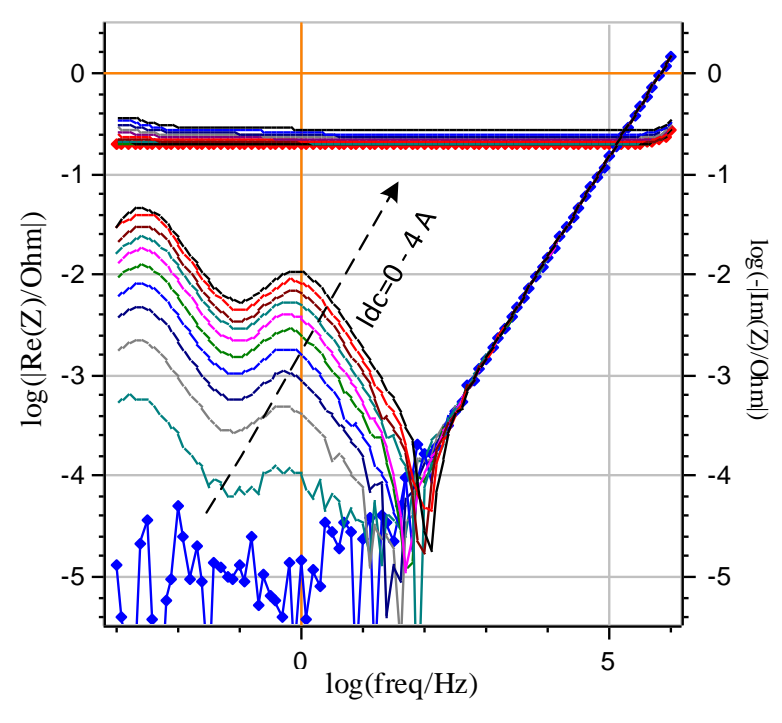

Figure 12. $\operatorname{Re}(Z)$ and $\operatorname{Im}(Z)$ of the silver wire inside the alumina tube. The same experimental conditions as pointed in Figure 11.

0 A ... 4 A with steps 0.4 A, and the test signal was $I a c=100 \mathrm{~mA}$ (it satisfies the low signal approach).

Figure 12 and Figure 13 show the results of the silver wire inside alumina and Teflon tubes, respectively.

A comparison between these experiments in the form of a Nyquist plot at one of the biases (at $I d c=2 \mathrm{~A}$ ) is shown in Figure 14.

It can be seen with the naked eye that an open wire has one time constant while the covered conductors have two time constants. Yet Teflon covering shows more overlapping and more distributed impedance spectra.

The fitting of the low-frequency part of the data $(100 \mathrm{~Hz} \ldots$ $1 \mathrm{mHz}$ ) using the equivalent $R C$-circuits is also presented in Figure 14. The fitting results are summarised in Table 2 . The indexes in the equivalent circuits in the table have the following meaning: $\mathrm{p}$ - parallel connection and $\mathrm{s}$ - serial connection.

Evaluating the fitting results, we can say that these objects are quite satisfactorily approximated by models utilising lumped

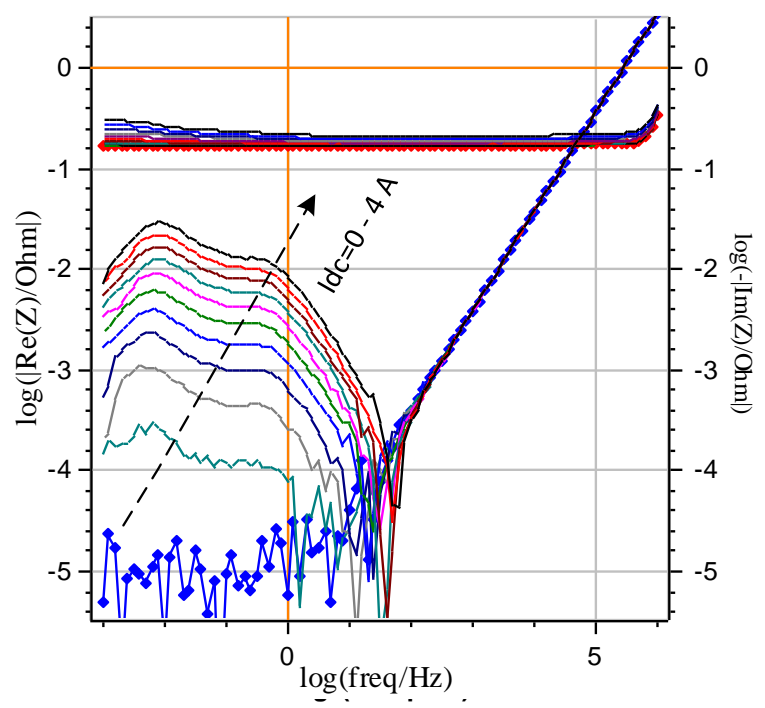

Figure 13. $\operatorname{Re}(Z)$ and $\operatorname{Im}(Z)$ of the silver wire inside the Teflon tube. The same experimental conditions as pointed in Figure 11.

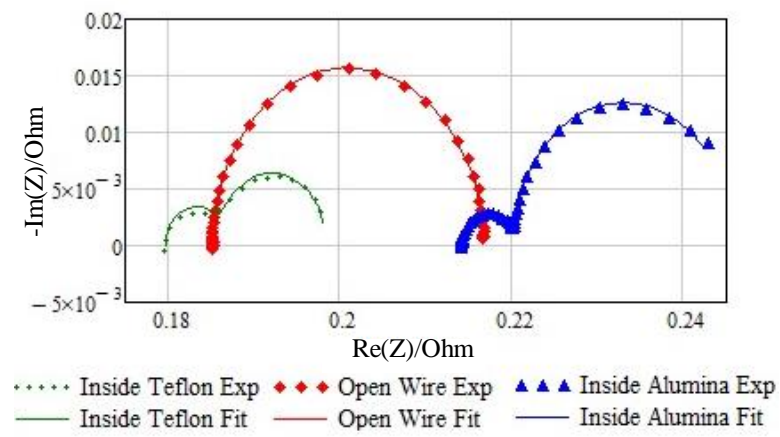

Figure 14. Nyquist plot of experimental and fitted data of non-covered silver wire, the wire inside alumina, and the wire inside Teflon. Low frequency part of data: $100 \mathrm{~Hz} \ldots 1 \mathrm{mHz}$ at the bias $/ d c=2 \mathrm{~A}$.

elements. A better result can be obtained for the case of a conductor surrounded by Teflon using the Gaussian distribution function, convolved into impedance [14]. But in this case, to demonstrate the $Z_{\mathrm{BI}}$-effect when exposed by the covering, this is not essential.

A simple calculation of the ratios of the time constants $(\tau=$ RC) taken from Table 2 gives the following values: $\tau 2 / \tau 1=247$ in the case of alumina covering and $\tau 2 / \tau 1=40$ in the case of Teflon covering.

Let us cite as an example the behaviour of an object exhibiting an inductive nature of $\mathrm{Z}_{\mathrm{BI}}$-effect also in a free and covered state. Figure 15 shows resulting Nyquist graphs of a graphite rod at bias $I d c=0.9 \mathrm{~A}$ and the test signal $I a c=100 \mathrm{~mA}$ in the open state and covered with alumina and Teflon tubes.

It is important to emphasise that the imaginary part has the opposite sign compared to the previous graphs of the silver wire.

The fitting results are summarised in Table 3 . The ratio of the two time constants $(\tau=L / R)$ for the graphite rod covered by alumina is $\tau 2 / \tau 1=284$. In the case of Teflon covering, the ratio is $\tau 2 / \tau 1=22$. The difference in the ratios of the time constants is similar to that found earlier in experiments with silver wire covered with the same materials.

\section{CHECK OF THE DATA CONSISTENCY}

Current-voltage characteristics were acquired on the same samples to check a data set for internal consistency. A sweep rate of $1 \mathrm{mV} / \mathrm{s}$ which is reasonable to our low frequency $0.1 \mathrm{~Hz}$ (in the first studies) was selected. This speed allows getting quasistatic characteristics. Static and differential parameters, namely resistances, were calculated and compared with parameters obtained from impedance measurements.

Table 2. Fitting results of non-covered silver wire, the wire inside alumina, and the wire inside Teflon (according to Figure 14).

\begin{tabular}{lll}
\hline SUT & Equivalent circuit & $\begin{array}{l}\text { Fit parameters: } \\
\boldsymbol{R} / \text { Ohm; } \boldsymbol{C} / \mathbf{F}\end{array}$ \\
\hline $\begin{array}{l}\text { Open } \\
\text { wire }\end{array}$ & $(R 1 p+C 1 p) s+$ & $\begin{array}{l}R 1 p=0.032 ; C 1 p=64.27 \\
R s=0.185\end{array}$ \\
\hline $\begin{array}{l}\text { Wire } \\
\text { inside alumina }\end{array}$ & $(R 1 p+C 1 p) s+$ & $R 1 p=6.0 \mathrm{e}-3 ; C 1 p=42.3$ \\
& $R s+C 2 p) s+$ & $R 2 p=0.025 ; C 2 p=2.48 \mathrm{e} 3$ \\
Wire & $(R 1 p+C 1 p) s+$ & $R 1 p=0.214$ \\
inside Teflon & $(R 2 p+C 2 p) s+$ & $R 2 p=0.013 ; C 2 p=1.86 \mathrm{e} 3$ \\
& $R s$ & $R s=0.179$ \\
\hline
\end{tabular}


Table 3. Fitting results of the non-covered graphite rod, the rod inside alumina, and the rod inside Teflon (according to Figure 15)

\begin{tabular}{|c|c|c|}
\hline SUT & Equivalent circuit & $\begin{array}{l}\text { Fit parameters: } \\
R / O \mathrm{Om} ; \mathrm{L} / \mathrm{H}\end{array}$ \\
\hline $\begin{array}{l}\text { Open } \\
\text { rod }\end{array}$ & $\begin{array}{l}(R 1 p+L 1 p) s+ \\
R s\end{array}$ & $\begin{array}{l}R 1 p=0.160 ; L 1 p=0.343 \\
R s=1.09\end{array}$ \\
\hline $\begin{array}{l}\text { Rod } \\
\text { inside alumina }\end{array}$ & $\begin{array}{l}(R 1 p+L 1 p) s+ \\
(R 2 p+L 2 p) s+ \\
R s\end{array}$ & $\begin{array}{l}R 1 p=0.01 ; L 1 p=1.33 \mathrm{e}-3 \\
R 2 p=0.121 ; L 2 p=4.572 \\
R s=1.128\end{array}$ \\
\hline $\begin{array}{l}\text { Rod } \\
\text { inside Teflon }\end{array}$ & $\begin{array}{l}(R 1 p+L 1 p) s+ \\
(R 2 p+L 2 p) s+ \\
R s\end{array}$ & $\begin{array}{l}R 1 p=0.041 ; L 1 p=0.027 \\
R 2 p=0.095 ; L 2 p=1.38 \\
R s=1.041\end{array}$ \\
\hline
\end{tabular}

The $I$ - $V$ characteristics of a silver sample are shown in Figure 16. The parameters of the sample under test correspond to the parameters indicated in Figure 3. The setup for $I-V$ measurements was identical to the setup for impedance measurements.

Figure 16 represents $I-V$ curve, static resistance $R$ stat $=$ $V d c / I d c$ and differential resistance $R$ diff $=\mathrm{d}(V d c) / \mathrm{d}(I d c)$. Parabolic spline interpolation was used for analytical differentiation. A fairly good accordance was obtained between the model parameters extracted from impedance measurements and the parameters calculated from the current-voltage characteristics. The parameter Rs extracted from the impedance $Z_{\mathrm{BI}}$ and the Rstat extracted from the $I-V$ well fit each other in an error of not more than $0.3 \%$. The total resistance $R$ sum $=R s+R_{1}$ found from the impedance measurements corresponds to the resistance Rdiff calculated from the $I-V$ (Figure 16). As an example, the bias point $V d c=0.72 \mathrm{~V}$ was taken in Figure 16 for indicating these correlations. It is corresponding to this bias point in Figure 6, Figure 8 and Figure 9.

The dependence of power dissipation $P_{\mathrm{i}}+\Delta P$ on the influence of bias and the test signal at an operating point $i$ will have the form:

$$
P_{i}+\Delta P=\left(V_{i}+\Delta V\right) \cdot\left(I_{i}+\Delta I\right),
$$

therefore, changes in power due to only the test signal is determined as

$$
\Delta P=V_{i} \cdot \Delta I+I_{i} \cdot \Delta V+\Delta V \cdot \Delta I,
$$

where: $V_{\mathrm{i}}, I_{\mathrm{i}}$ - voltage and current at a working point and $\Delta V, \Delta I$ - amplitudes of voltage and current of the test signal.

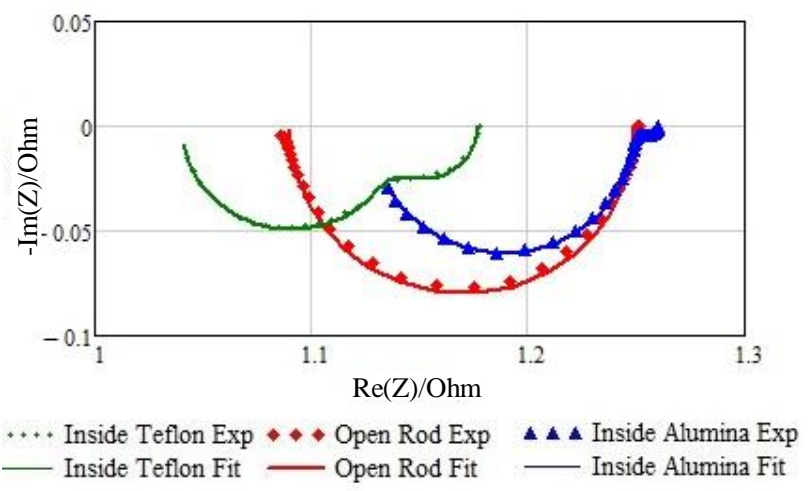

Figure 15. Nyquist plot of experimental and fitted data of non-covered graphite rod, the rod inside alumina, and the rod inside Teflon. Low frequency part of data $100 \mathrm{~Hz} \ldots 1 \mathrm{mHz}$ at the bias $/ d c=0.9 \mathrm{~A}$.

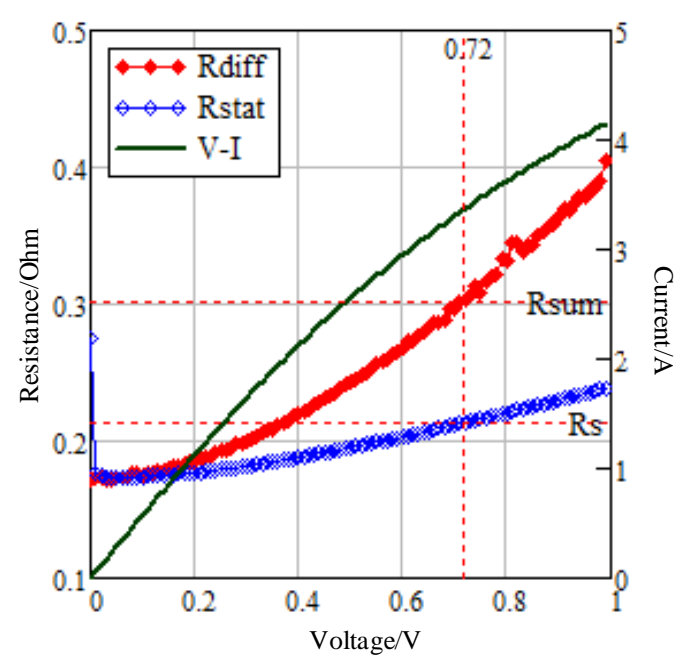

Figure 16. I-V curve; static and differential resistance curves of silver wire with the same dimensions as in Figure 3.

From (4), it can be noticed that with increasing bias the dissipated power caused by the test signal will increase. Hence, the temperature variation will increase, which will lead to an increase in a change of the resistivity by an influence of the test signal. This is an explanation of the magnification of the $\mathrm{Z}_{\mathrm{BI}}$-effect at all the experiments with increasing a bias.

The most mysterious case is the presence of both types of reactivity in experiments with alloys (Figure 4 and Figure 7). It required an additional consistency check through an independent method. For these purposes, current-voltage characteristics with different sweep rates were used. The sweep rates were chosen to match the transient point around $0.5 \mathrm{~Hz}$ (Figure 7). The corresponding graphs of the $I-V$ characteristics are shown in Figure 17. The setup was the same as for impedance measurements.

Figure 18 shows the graphs of the calculated values of the static resistances Rstat obtained from the $I-V$ characteristics with different sweep rates (Figure 17).

Now we can see that, in the vicinity of $5.3 \mathrm{~V}$ bias voltage, the static resistance changes the trend from decreasing its value with increasing a bias at the sweep $0.1 \mathrm{mV} / \mathrm{s}$ to increasing its value with increasing a bias at the sweep $100 \mathrm{mV} / \mathrm{s}$. The sign of the

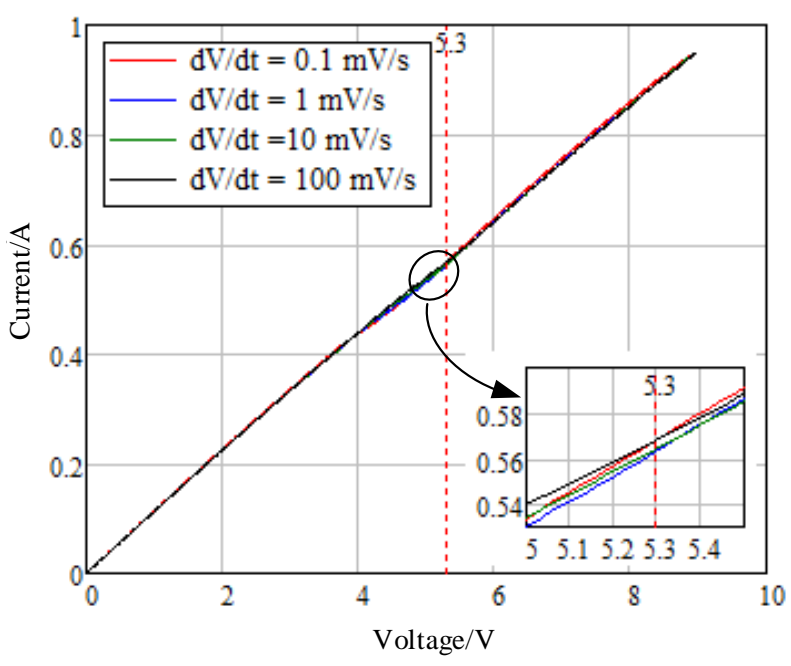

Figure $17 . \mathrm{I}-\mathrm{V}$ curves with voltage sweeps: $0.1 ; 1 ; 10$ and $100 \mathrm{mV} / \mathrm{s}$. Nichrome wire, the same dimensions as pointed in Figure 4. 


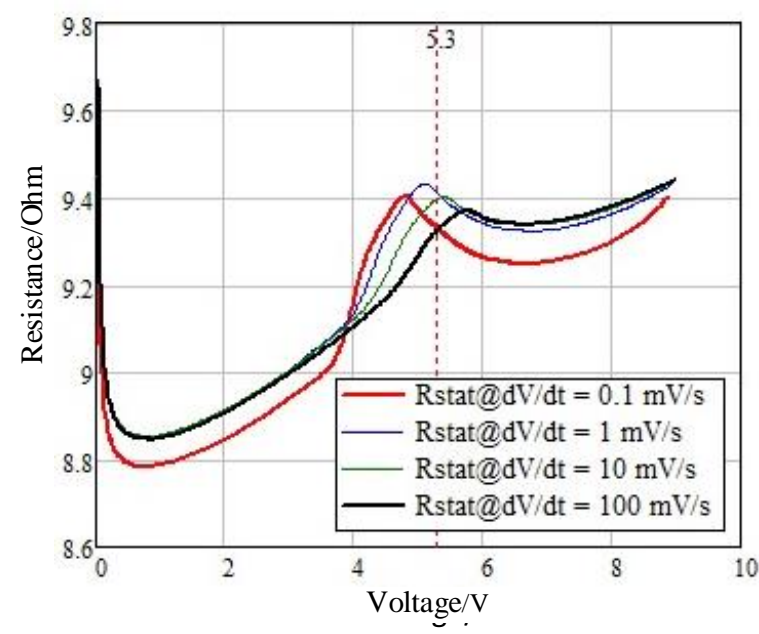

Figure 18. Static resistances calculated from the $I-V$ curves represented in Figure 17.

differential resistance, which is the essence of impedance measurements, will change accordingly. Thus, there is a consistency between measurements in the frequency domain (Figure 7) and time domain (Figure 18).

Several temperature studies were carried out to check the data consistency. The platinum conductor was heated by a separate heating element at various biases.

Figure 19 shows the results of the LF part of data $100 \mathrm{~Hz}-$ $0.1 \mathrm{~Hz}$ at different values of temperature controller: at room temperature, at $165 \mathrm{C}^{\circ}$, and $265 \mathrm{C}^{\circ}$. The actual temperature of the wire can be calculated considering the resistivity of the wire - via real part of impedance at frequency $100 \mathrm{~Hz}$ or using DC measurement. For qualitative analysis, knowing the actual temperature of the wire is not essential in our case.

The main results of this experiment are as follows. We see that the $\mathrm{Z}_{\mathrm{BI}}$-effect does not occur in the absence of bias at any temperature. At the same time, this effect takes place in the presence of a bias at any temperature. It is also noticeable that the external temperature additively shifts the values of the real impedance component, almost without affecting the imaginary one.

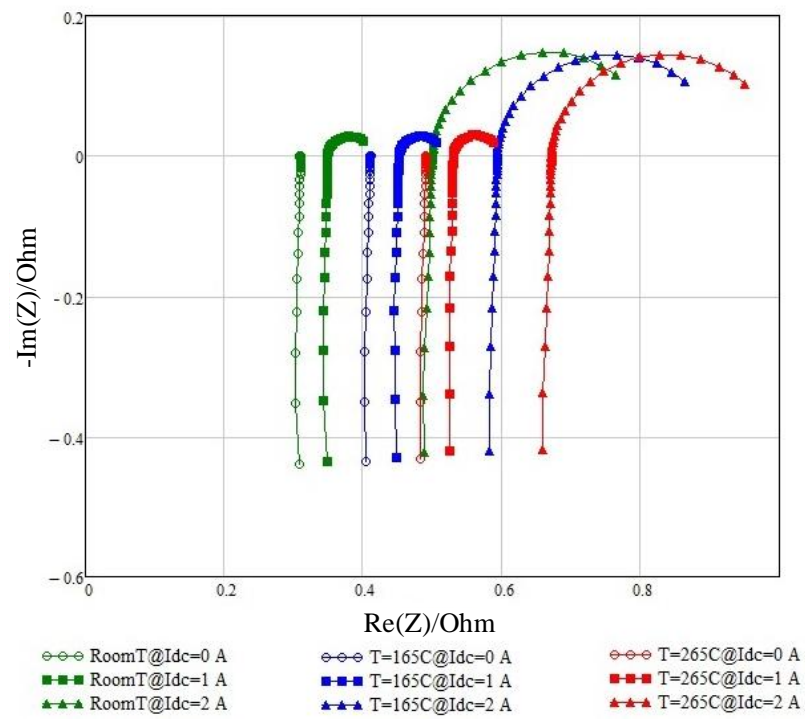

Figure 19. Nyquist plot of platinum wire at different temperatures and biases; length $83 \mathrm{~mm}$ and diameter $0.2 \mathrm{~mm}$; frequency range $100 \mathrm{~Hz} \ldots 0.1 \mathrm{~Hz}$.
A good illustration in this graph is the occasion when, in the case of room temperature and bias $I d c=2 \mathrm{~A}$, the actual conductor temperature is practically identical with the case when the temperature controller value shows $265 \mathrm{C}^{\circ}$ without bias applied to the conductor $(I d c=0 \mathrm{~A})$.

\section{DISCUSSIONS}

The impedance of quite ordinary current conductors under bias in the low-frequency region demonstrates remarkable properties - named as $Z_{\mathrm{BI}}$-effect. The term "current-carrying conductor" refers to extended conductors designed to carry electric current. The research that is outlined in this article is also relevant to other types of objects. These can be conductive or semiconductive materials of various compositions and shapes. An example relates to experiments carried out with thermistors (they are out of the scope of the paper).

The $Z_{\mathrm{BI}}$-effect is counter-intuitive. It was natural to assume that the measurement of the impedance of objects at the lowest frequency brings it closer to the measurement result at direct current. This is what happens in the absence of bias. Yet, if a bias is applied to an object the feeling deceives. It looks paradoxical, but the infra-low frequency is not an asymptotic approximation to DC when measuring the impedance of a conductor under bias.

Now, the question of how to explain the occurrence of such significant reactive elements in the impedance models of the studied objects. In particular, the capacitance of pure metals reaches the order of farads (Figure 8). The inductance of graphite rods reaches about hundreds of millihenry (Figure 10). In reality, of course, such reactance does not exist in the studied objects. This phenomenon may be called as a "phantom" reactance.

This effect can be explained considering two necessary properties of the studied objects. The first is nonlinearity and the second one is inertia. The nonlinearity of current conductors is the second kind of nonlinearity (indirect). This property distinguishes them from objects of the first kind of nonlinearity (direct), such as $\mathrm{p}-\mathrm{n}$ junctions or Schottky diodes.

In the case of the first kind of nonlinearity, the nonlinearity reveals itself directly, without any delay. For the second kind of nonlinearity, it manifests itself due to a resistivity dependence on temperature. This is a factor of the studied material. The nonlinearity of the second kind has a significant delay. The bias sets a specific operating point. The test signal acts in the vicinity of this point. No matter how small the test signal is, it will change the temperature of the investigated object in the locality of the operating point with a certain delay. Consequently, the resistance of the material under investigation will cyclically change with the test signal.

Eventually, the resistance is modulated by the test signal. The difference between the phase of this modulation and the phase of the acting test signal determines the occurrence of phantom reactance. If the investigated object has a PTC property, a capacitive reactance arises. This behavior is typical for pure metals or PTC thermistors. If the object under study has an NTC feature, then an inductive reactance appears. It is specific, for example, to graphite and NTC thermistors.

In terms of electrical measurements, impedance is properly defined only for systems satisfying stationarity [6]. In our case, we have a dynamic structure with one exception - the system changes cyclically and synchronously with the influence of the test signal. The amplitude and phase response depends on the frequency of the test signal. A purely active resistance, which changes are synchronously according to the test signal, but with 
a different phase relative to the test signal, generates a reaction that looks like a complex resistance. As a result, a complex value will be estimated during the measurements as an impedance of the studied object.

Successive experiments revealed a significant feature. It turned out that the time constant following from the $Z_{\mathrm{BI}}$-effect's model ( $\tau=R C$ for PTC objects and $\tau=L / R$ for NTC objects) weakly depends on the applied bias. It is reasonable to assume that these time constants related to the time constant of the heat exchange between the object under study and the environment (air in our initial study case). Studies using a various covering of current-carrying conductors support this hypothesis. There are two time constants (Figure 12 and Figure 13). The first of which is apparently determined by the thermal properties of the covering and the thermal interaction between the conductor and the covering. The second time constant is determined by the thermal interaction between the covering and the environment.

An accurate description of thermal processes requires special knowledge and is beyond the scope of this article. However, it seems possible that the discovered effect could allow to develop specialised sensors to assess the thermal conductivity of various materials. This approach may be an alternative to the methods described in [15], [16].

The experimental results obtained on the nichrome alloy motivates the appearance of additional ideas. In particular, both capacitive and inductive reactive components are observed at a bias $V d c=5.3 \mathrm{~V}$ (Figure 7) and in the areas close to it (Figure 4) depending on the frequency of the test signal. Specifically, for Figure 7 capacitive nature takes place in the frequency range of $10 \mathrm{~Hz}$ to $0.5 \mathrm{~Hz}$, and inductive nature takes place in the range of $0.5 \mathrm{~Hz}$ to $0.1 \mathrm{~Hz}$. Such effects are possible if we assume that the temperature coefficient of resistance (TCR) has dynamic properties. In other words, the TCR changes its character depending on the rate of temperature change. In turn, the rate of temperature variations at the selected operating point will be related to the frequency of the test signal. Therefore, in the higher frequency range will be observed a PTC-feature, in the lower frequency range will be observed an NTC-feature (Figure 7). This assumption was confirmed using $I-V$ experiments with different sweep rates (Figure 17 and Figure 18). It looks like impedance spectroscopy may provide a more sensitive tool for assessing the dynamic properties of the TCR.

Since the dynamic properties of the TCR will depend on the composition of the objects under study, there is a potential possibility of indirect composition estimation by checking the moment of the TCR sign change utilising impedance spectroscopy.

The revealed new properties (i.e., the possibility of evaluating the thermal conductivity and estimating the composition of the material by dynamic TCR, arising from the discovered $Z_{\mathrm{BI}}-$ effect $t$ may represent a significant contribution to the scientific and technical community, in particular, in the development of the theory and practice of impedance spectroscopy of objects that cyclically change their parameters synchronously with the test signal. Understanding the nature of this effect can foster the development of a new type of instruments in various fields and various scientific institutions.

\section{CONCLUSIONS}

In this work, the phenomenon of bias-induced impedance was described. This effect was most evident in the low frequency spectra of the reactive part of the impedance. The manifestation of the different nature of this issue was shown experimentally. The $Z_{\mathrm{BI}}$-effect may be capacitive, inductive, or complex, which includes both types of reactance. The nature of the reactance depends on the type of test material. Pure metals showed capacitive reactance. Graphite rods showed inductive reactance. The alloys showed reactance of both types depending on the level of bias. The investigated objects can be attributed to the inertial nonlinear resistances.

The $Z_{\mathrm{BI}-\text { effect }}$ is caused by the thermal interaction between the conductor and the environment under the superposition of the bias combined with a test signal.

Relatively simple equivalent circuits were found to describe the experimental data. Additional studies should be undertaken to better understand the behaviour of alloys and other composites under the bias, especially unexpected dynamic TCR properties.

New possibilities arise for assessing the thermal conductivity of various materials. This requires the synthesis of knowledge in the fields of electrical and thermal measurements and the construction of specialised sensor devices.

\section{REFERENCES}

[1] E. H. Nikollian, J. R. Brews, MOS Physics and Technology, Wiley, New York, 1982. ISBN-13: 978-0471430797

[2] S. Taibl, G. Fafilek, J. Fleig, Impedance spectra of Fe-doped SrTiO3 thin films upon bias voltage: Inductive loops as a trace of ion motion, Nanoscale. 8 (2016), pp.13954-13966. DOI: $10.1039 / \mathrm{c} 6 \mathrm{nr} 00814 \mathrm{c}$

[3] N. Kumar, E. A. Patterson, T. Frömling, D. P. Cann, DC-bias dependent impedance spectroscopy of $\mathrm{BaTiO}_{-}$ Bi(Zn1/2Ti1/2)O3 ceramics, J. Mater. Chem. C. 4 (2016), pp. $1782-1786$. DOI: $10.1039 / \mathrm{c} 5 \mathrm{tc} 04247 \mathrm{i}$

[4] Z. B. Stoynov, B. M. Grafov, B. S. Savova-Stoynov, V. V. Elkin, Electrochemical impedance, Nauka, Moscow, 1991. ISBN: 5-02001945-3

[5] S. Baltianski, Low frequency bias-induced impedance, 24th IMEKO TC4 Int. Symp. 22nd Int. Work. ADC and DAC Model. Test, Palermo, Italy, 14-16 September 2020, pp 423-428. Online [Accessed 18 June 2021] https://www.imeko.org/publications/tc4-2020/IMEKO-TC42020-79.pdf

[6] E. Barsoukov, J. R. Macdonald (Eds.), Impedance spectroscopy, theory, experiment, and applications, 2nd Ed., New Jersey, John Wiley \& Sons. Inc., 2005. ISBN: 978-0-471-64749-2

[7] M.E. Orazem (Eds.), B. Tribollet, Electrochemical Impedance Spectroscopy, 2nd Ed., New Jersey, John Wiley \& Sons, Inc., 2008. ISBN:9780470041406

[8] A. Lasia, Electrochemical impedance spectroscopy and its applications, Electrochem. Impedance Spectrosc. Its Appl. 9781461489337 (2014) 1-367.

DOI: $\underline{10.1007 / 978-1-4614-8933-7}$

[9] F. W. Grover, Inductance calculations: Working formulas and tables (Dover Phoenix Editions) Hardcover - March 29, 2004. ISBN-10: 0486495779

[10] N. Murer, Installation and configuration manual for VMP-300based instruments and boosters. Online [Accessed 05 June 2021]. https://www.biologic.net/documents/vmp300-based-manuals/

[11] P. Baranov, V. Borikov, V. Ivanova, B. B. Duc, S. Uchaikin, C. Y. Liu, Lock-in amplifier with a high common-mode rejection ratio in the range of 0.02 to $100 \mathrm{kHz}$, Acta IMEKO 8(1) (2019), pp. 103-110. DOI: $10.21014 /$ acta imeko.v811.672

[12] S. S. Baltyanskii, Measuring the parameters of physical objects by identifying electrical models, Meas. Tech. 43 (2000), pp. 763-769. DOI: $\underline{10.1023 / A: 1026645722396}$ 
[13] F. M. Janeiro, P. M. Ramos, Gene expression programming and genetic algorithms in impedance circuit identification, Acta IMEKO 1(1) (2012), pp. 19-25.

DOI: $10.21014 /$ acta imeko.v1i1.16

[14] S. Baltianski, Impedance spectroscopy: Separation and asymptotic model interpretation, XXI IMEKO World Congr. Measurement Res. Ind., Prague, Czech Republic, 30 August - 04 September 2015, pp. 492-497. Online [Accessed 05 June 2021].

https://www.imeko.org/publications/wc-2015/IMEKO-WC2015-TC4-101.pdf
[15] E. Barsoukov, J. H. Jang, H. Lee, Thermal impedance spectroscopy for Li-ion batteries using heat-pulse response analysis, J. Power Sources 109 (2002), pp. 313-320. DOI: $10.1016 /$ S0378-7753(02)00080-0

[16] M. Swierczynski, D. I. Stroe, T. Stanciu, S. K. Kær, Electrothermal impedance spectroscopy as a cost efficient method for determining thermal parameters of lithium ion batteries: Prospects, measurement methods and the state of knowledge, J. Clean. Prod. 155 (2017), pp. 63-71. DOI: $10.1016 /$ i.jclepro.2016.09.109 